\title{
Escape the bear and fall to the lion: the impact of avoidance availability on threat acquisition and extinction
}

Article

Accepted Version

Creative Commons: Attribution-Noncommercial-No Derivative Works 4.0

Morriss, J., Chapman, C., Tomlinson, S. and Van Reekum, C. M. (2018) Escape the bear and fall to the lion: the impact of avoidance availability on threat acquisition and extinction. Biological Psychology, 138. pp. 73-80. ISSN 0301-0511 doi: https://doi.org/10.1016/j.biopsycho.2018.08.017 Available at https://centaur.reading.ac.uk/79083/

It is advisable to refer to the publisher's version if you intend to cite from the work. See Guidance on citing.

To link to this article DOI: http://dx.doi.org/10.1016/j.biopsycho.2018.08.017

Publisher: Elsevier

All outputs in CentAUR are protected by Intellectual Property Rights law, including copyright law. Copyright and IPR is retained by the creators or other copyright holders. Terms and conditions for use of this material are defined in the End User Agreement.

www.reading.ac.uk/centaur 
Central Archive at the University of Reading

Reading's research outputs online 
Escape the bear and fall to the lion: The impact of avoidance availability on threat acquisition and extinction

Jayne Morriss ${ }^{*}$, Catherine Chapman, Susan Tomlinson, and Carien M. van Reekum

Centre for Integrative Neuroscience and Neurodynamics

School of Psychology and Clinical Language Sciences

University of Reading

Reading

UK

${ }^{*}$ Correspondence:

Jayne Morriss

Centre for Integrative Neuroscience and Neurodynamics

School of Psychology and Clinical Language Sciences

University of Reading

Earley Gate, Whiteknights Campus

RG6 6AH Reading

United Kingdom

j.e.morriss@reading.ac.uk 


\begin{abstract}
Pervasive avoidance behaviour is a core feature of anxiety disorders. However, little is known about how the availability of avoidance modulates learned threat responding. To assess this question, we recorded avoidance behaviour, electrodermal activity and expectancy ratings in 53 healthy participants during an associative learning paradigm with embedded unavoidable and avoidable trials. When avoidance was available, we observed greater avoidance behaviour for threat versus safety cues, as well as reduced differential skin conductance responses for unavoidable threat versus safety cues. When avoidance was unavailable, as during the extinction phase, we observed sustained differential skin conductance responses for threat versus safety cues. For all phases, we found greater expectancy ratings for threat versus safe cues. Furthermore, greater avoidance behaviour predicted larger differential skin conductance responses to threat versus safety cues during extinction. Overall, the results show that the conditioned response is attenuated during situations where avoidance is available, but it recovers when avoidance is unavailable, subsequently prolonging threat extinction.
\end{abstract}

Keywords: Threat, Conditioning, Extinction, Avoidance, Anxiety 
The ability to discriminate between threat and safety is crucial for survival. Through classic threat conditioning, an initially neutral cue (conditioned stimulus [CS], such as a visual shape) comes to be associated with an aversive outcome (unconditioned stimulus [US], e.g. shock, loud tone). Repeated presentations of a neutral cue with an aversive outcome can result in defensive responses to the neutral cue alone (conditioned response). This learned association can be mitigated by repeatedly presenting the learned threat cue without the aversive outcome, a process known as extinction (Milad \& Quirk, 2012). Avoidance learning has been shown to alter the course of extinction or even block extinction from occurring altogether (Andreatta, Michelmann, Pauli, \& Hewig, 2017; Lovibond, Mitchell, Minard, Brady, \& Menzies, 2009; Vervliet \& Indekeu, 2015; Xia, Dymond, Lloyd, \& Vervliet, 2017).

Avoidance learning is a type of instrumental learning where the US is prevented through an action. Avoidance learning is commonly measured by a button press in response to the onset of a CS, in order to avoid a paired US (Boeke, Moscarello, LeDoux, Phelps, \& Hartley, 2017; Boyle, Roche, Dymond, \& Hermans, 2015; Delgado, Jou, LeDoux, \& Phelps, 2009; Lommen, Engelhard, \& van den Hout, 2010; Lovibond et al., 2009; Lovibond, Saunders, Weidemann, \& Mitchell, 2008; Vervliet \& Indekeu, 2015; Xia et al., 2017). However, other studies have also measured avoidance learning through examining participants' choices to avoid a paired US via a detour in gaming tasks (Rattel, Miedl, Blechert, \& Wilhelm, 2017; van Meurs, Wiggert, Wicker, \& Lissek, 2014), and via deck selection in a gambling task (Pittig, Brand, Pawlikowski, \& Alpers, 2014). By avoiding the aversive outcome (US, e.g. shock or tone), the likelihood of threat is reduced. The two-factor theory posits 
that avoidant behaviour is negatively reinforced through feelings of reward and relief (Mowrer, 1951, 1956).

Vervliet and Indekeu (2015) found that avoidance learning resulted in reduced extinction on the same day, indexed by greater skin conductance response and ratings of shock expectancy to learned threat vs. safety cues. Similarly, Xia, Dymond, Lloyd and Vervliet (2017) found that higher pairing rates between the CS and US (e.g. 50-100\%), compared to lower pairing rates (e.g. 0-25\%), during acquisition resulted in greater behavioural avoidance and reduced extinction on the same day in shock expectancy ratings. However, a handful of studies on rodents and humans have also shown that previous experience of avoidance can promote extinction (Baratta et al., 2007; Boeke et al., 2017; Cain \& LeDoux, 2007). In these experiments, the avoidance is considered active, as both rodents and humans have to learn how to avoid the threat (i.e. moving to another chamber or correctly changing the location of a circle in a grid). In the rodent studies, learning how to actively avoid a shock facilitated extinction during the same avoidance learning context (Cain \& LeDoux, 2007) and in a different threat learning context days later (Baratta et al., 2007). Boeke et al. (2017) observed that active avoidance in humans led to reduced threat responding during a next day extinction (or retrieval) phase. The differences in extinction findings in the avoidance learning literature may be underpinned by instruction of avoidance (explicitly instructed what action to make versus explicitly instructed to learn the correct action to make), the availability of avoidance (e.g. escapable, inescapable), the time frame by which extinction is assessed (e.g. same day, next day or over the course of a week) and the cost of avoidance (e.g. type of US, loss of points, type of rewards). 
The mixed findings in the literature notwithstanding, avoidance learning has been highlighted as a potentially important mechanism in anxiety disorder pathology. Engaging in avoidance may provide greater feelings of reward and relief in individuals who are more susceptible to anxiety (Mowrer, 1951, 1956), such as those with low distress tolerance (Vervliet, Lange, \& Milad, 2017). The effect of avoidance on reduced extinction is thought to occur because avoidance leaves the learned threat association unchallenged. The findings above suggest it may be adaptive to avoid some situations associated with threat but continued avoidance may hinder opportunities for learning about situations associated with safety (LeDoux, Moscarello, Sears, \& Campese, 2017). This argument has been incorporated in contemporary models of pervasive avoidance behaviour observed in anxiety disorders (Dymond \& Roche, 2009; Krypotos, Effting, Kindt, \& Beckers, 2015).

Commonly, prior work on avoidance learning has presented participants with the possibility of avoiding all trials within the avoidance phase (but see Rattel et al, 2017 who included unavoidable trials). However, everyday life is more complex, lending some contexts as more avoidable than others. The availability of avoidance may modulate how learned threat associations are expressed and maintained. For example, being able to avoid the US some of the time will likely result in quelled responding to unavoidable learned threat cues, due to decreased US reinforcement. The availability of avoidance therefore may provide feelings of relief, as the overall likelihood of threat is reduced. However, when avoidance is unavailable, the learned threat association is likely to return as there is greater chance of threat. Furthermore, a history of greater avoidance behaviour may predict a stronger return of the learned threat association. 
In the current study we attempted to address whether having availability of avoidance changes the expression of learned threat during acquisition and extinction. Here we expanded the experimental designs of past work (Vervliet \& Indekeu, 2015; Xia et al., 2017) by including unavoidable trials within the avoidance phases to allow for the assessment of the conditioned response measured via skin conductance response. Furthermore, we extended work by Rattel et al. (2017) by allowing participants to freely choose to avoid without manipulating avoidance costs. We used a $50 \%$ pairing rate in the acquisition phase as this is known to prolong conditioning (Jenkins \& Stanley Jr, 1950) and produce more avoidant behaviour (Xia et al., 2017). The $50 \%$ pairing rate was then continued in the subsequent acquisition avoidance phase, where participants were presented with a mixture of avoidable and unavoidable trials. During this phase, we assessed whether the availability of avoidance could change the expression of the conditioned responses on unavoidable trials. Following this, we included an extinction phase where all trials were unavoidable, to assess how previous availability of avoidance leads to a return of the conditioned response. Lastly, we presented an extinction-avoid phase with avoidable and unavoidable trials. Within our adapted experimental design, participants were able to avoid the learned threat and safe trials by pressing a button in both of the avoidance phases.

Throughout the task, we recorded expectancy ratings, skin conductance and behavioural avoidance responding measured through button press. Firstly, we predicted greater expectancy ratings and skin conductance to the learned threat vs. safe cues during acquisition. Secondly, we predicted larger expectancy ratings and greater behavioural avoidance to the learned threat vs safe cues during the two 
avoidance phases (acquisition avoid; extinction avoid). Thirdly, we predicted reduced skin conductance to the unavoidable learned threat trials during the avoidance phases, due to lack of US reinforcement. Furthermore, in line with previous work we predicted that, following avoidance learning, there will be evidence of extinction resistance, such that there will be greater expectancy ratings and skin conductance to the learned threat vs. safe cues during extinction. Lastly, we expected individual differences in the total rate of avoidance behaviour during acquisition avoid, as measured by the number of button presses to both the learned threat and safe cues, to predict the extent of conditioned responding during extinction e.g. avoiding more trials would equate to greater extinction resistance.

\section{Method}

\section{Participants}

For this experiment 53 students took part (mean age $=19.74 ; 48$ females $\& 5$ males). This sample size was based on an a priori sample size calculation for a repeated measures ANOVA (F-test with power $(1-\beta)$ set at $0.8, \alpha=.05$, effect size of 0.22 , number of groups set to 1 , number of measurements set to 2 (CS+, CS-) and correlation estimate of 0.4 between measures). The value for the effect size $(F(1,18)=4.93, p<0.05, \eta 2=0.22)$ is based on the reported main effect of CS for skin conductance response during extinction after avoidance learning (Vervliet \& Indekeu, 2015). From this power analysis a minimum sample of 51 participants was recommended. All participants had normal or corrected to normal vision. Participants provided written informed consent and received course credit for their participation. 
Participants were recruited through the University of Reading Psychology Panel. The procedure was approved by the University of Reading Ethics Committee.

There was no exclusion criteria. The data of 2 participants were removed through a programming error and 1 participant was removed due to a physiology recording error, leaving 50 subjects.

\section{Procedure}

Participants arrived at the laboratory and were informed on the procedures of the experiment. Firstly, participants were taken to the testing booth and given a consent form to sign as an agreement to take part in the study. Secondly, to assess anxious disposition we asked participants to complete a series of questionnaires (see Supplementary Material) presented on a computer in the testing booth (not reported here). Next, physiological sensors were attached to the participants' nondominant hand. Participants were simply instructed to: (1) maintain attention to the task by looking and listening to the coloured squares and sounds presented, (2) respond to the expectancy rating scales that followed each phase (see "Conditioning task" below for details) using the keyboard with their dominant hand, (3) avoid trials with a red symbol if they wanted using the left mouse button with their dominant hand, (4) to sit as still as possible. Participants were presented with a conditioning task on the computer, whilst electrodermal activity, interbeat interval and ratings were recorded. After the conditioning task, subjects were asked to rate the valence and arousal of the sound stimulus (US) using 9-point Likert scales ranging from 1 (Valence: very negative; Arousal: calm) to 9 (Valence: very positive; Arousal: excited). Altogether, the experiment took approx. 45 minutes. 


\section{Conditioning task}

The conditioning task was designed using E-Prime 2.0 software (Psychology Software Tools Ltd, Pittsburgh, PA). Visual stimuli were presented using a screen resolution of $800 \times 600$ with a 60 Hertz refresh rate. Participants sat at approximately $60 \mathrm{~cm}$ from the screen. Sound stimuli were presented through headphones.

Visual stimuli were light blue and yellow squares with $183 \times 183$ pixel dimensions that resulted in a visual angle of $5.78^{\circ} \times 9.73^{\circ}$. The aversive sound stimulus consisted of a fear inducing female scream (sound number 277) from the International Affective Digitized Sound battery (IADS-2) and which has been normatively rated as unpleasant $(M=1.63, S D=1.13)$ and arousing $(M=7.79, S D=$ 1.13) (Bradley \& Lang, 2007). We used Audacity 2.0.3 software (http://audacity.sourceforge.net/) to shorten the female scream to $1000 \mathrm{~ms}$ in length and to amplify the sound by $15 \mathrm{~dB}$, resulting in a $90 \mathrm{~dB}(+/-5 \mathrm{db})$ sound (used previously in (Morriss, Christakou, \& Van Reekum, 2015, 2016; Morriss, Macdonald, \& van Reekum, 2016). An audiometer was used before testing to standardise the sound volume across participants.

Habituation, acquisition, acquisition avoid, extinction and extinction avoid phases were presented in five separate blocks (see Fig. 1). At the start of the experiment participants were habituated to yellow and blue squares with no pairing. In acquisition, one of the squares was paired with the aversive $90 \mathrm{~dB}$ scream (unconditioned stimulus; US) $50 \%$ of the time (Conditioned Stimulus +; CS+), whilst the other square was presented alone (Conditioned Stimulus -; CS-). In acquisition avoid, the CS+ was still paired with the aversive $90 \mathrm{~dB}$ scream $50 \%$ of the time. The 
CS- remained unpaired. However, there were additional avoid trials (CS+ paired avoid, CS+ avoid, CS- avoid). During extinction, both stimuli were unpaired (CS+, CS-). In extinction avoid, both stimuli remained unpaired (CS+, CS-), but additional avoid trials were included (CS+ avoid, CS- avoid).

The avoid trials were signified by a red dot that appeared above the square at a central position, which allowed for avoidance through the click of the right mouse button (see Fig. 1). The CS and potential US disappeared once the right mouse button was clicked i.e. if participants received a CS+ avoid paired trial and clicked the right mouse button within the time of the CS presentation (4000 ms), then they could avoid the US. Therefore, the number of US exposures is variable dependent on participants' avoidance behaviour. The participants were instructed about the avoidance trials before the experiment started on the presentation computer. We used the following text, 'At some points in the experiment you will see a red dot like the one shown here. It will appear above a square. This means you will be given the option to avoid a trial by pressing the left mouse button.' After each rating period, participants were instructed on the presentation computer, 'please put your hand back on the mouse'.

The habituation phase consisted of 4 trials (2 CS+, 2 CS-). The acquisition phase consisted of 18 trials (6 CS+paired, 6 CS+unpaired and 6 CS-) and the acquisition avoid consisted of 36 trials (6 CS+paired, 6 CS+unpaired, 6 CS-, 6 CS+paired avoid, 6 CS+unpaired avoid and 6 CS- avoid). The extinction phase contained 32 trials (16 CS+, 16 CS-) and the extinction avoid phase contained 24 trials (6 CS+unpaired, 6 CS-, 6 CS+unpaired avoid and 6 CS- avoid). Experimental trials within the conditioning task were pseudo-randomised. The first trial of the 
acquisition and acquisition avoid phase started with a trial that was paired.

Thereafter all trials were randomised. Conditioning contingencies were counterbalanced, with half of the participants receiving the US with a blue square and the other half of participants receiving the US with a yellow square. Participants did not receive instructions about the contingency between the CS and US.

The presentation times of the task were: 4000 ms square, 1000 ms sound (co-terminated with the square), 6000 - 8800 ms blank screen (see Fig. 1). The expectancy rating scale asked whether participants expected a sound with each square separately, where the scale was 1 'Don't expect - 9 'Do expect'. Participants received expectancy rating scales on the computer at the end of the acquisition, acquisition avoid, extinction and extinction avoid phases.

Figure 1 about here.

\section{Avoidance behaviour and rating data scoring}

Avoidance behaviour and rating data were reduced for each subject by calculating their average responses for each experimental condition using the $\mathrm{E}$ Data Aid tool in E-Prime (Psychology Software Tools Ltd, Pittsburgh, PA). To make the frequency of avoidance for acquisition and extinction avoid phases comparable, we averaged frequency of avoidance across CS+ paired avoid and CS+ avoid trials in the acquisition avoid phase. For avoidance reaction time data see supplementary material.

Individual differences in total avoidance frequency were calculated separately for the acquisition avoid and extinction avoid phases. 


\section{Physiological acquisition and scoring}

Physiological recordings were obtained using AD Instruments (AD Instruments Ltd, Chalgrove, Oxfordshire) hardware and software. Electrodermal activity was measured with dry MLT116F silver/silver chloride bipolar finger electrodes that were attached to the distal phalanges of the index and middle fingers of the non-dominant hand (each electrode surface plate is $2 \mathrm{~cm}(\mathrm{I}) \times 2.5 \mathrm{~cm}(\mathrm{w})$ in size). To avoid excessive dryness due to soap or disinfectant the skin was not cleaned before the experiment. A low constant-voltage $A C$ excitation of $22 \mathrm{mV}_{\mathrm{rms}}$ at $75 \mathrm{~Hz}$ was passed through the electrodes, which were connected to a ML116 GSR Amp, and converted to DC before being digitized and stored. No filter was applied to the skin conductance response channel. Interbeat Interval (IBI) was measured using a MLT1010 Electric Pulse Transducer, which was connected to the participant's distal phalange of the ring finger. An ML138 Bio Amp connected to an ML870 PowerLab Unit Model 8/30 amplified the electrodermal and interbeat interval signals, which were digitized through a 16-bit A/D converter at $1000 \mathrm{~Hz}$. IBI signal was used only to identify movement artefacts and was not analyzed. The electrodermal signal was converted from volts to microSiemens using $A D$ Instruments software $(A D$ Instruments Ltd, Chalgrove, Oxfordshire).

Avoidance trials and CS+paired trials were discarded from the analysis, due to movement and sound confounds. All unavoidable trials were included, i.e. CS+ unpaired, CS-. Skin conductance responses (SCR) were scored when there was an increase of skin conductance exceeding 0.03 microSiemens. The amplitude of each SCR response was scored as the difference between the onset (foot of the peak) 
and offset (the peak). SCRs were counted if the SCR onset was within 0.5-3.5 seconds following CS onset. Trials with no SCRs were scored as zero (percentage of trials scored as zero during: Acquisition,52.5\%; Acqusition Avoid,63.3\%; Extinction, 68\%; Extinction Avoid, 70\%). SCR magnitudes were square root transformed to reduce skewness and were z-scored to control for interindividual differences in skin conductance responsiveness. Trials with motion artefacts, as identified by distortions in both electrodermal and IBI signals associated with movement were discarded from the analysis. $0.647 \%$ (44 out of 6800 ) trials were removed from the analysis due to movement artefacts. SCR magnitudes were calculated from remaining trials by averaging SCR square root transformed values and zeros for each condition. ${ }^{1}$

\section{Learning assessment}

To assess whether participants learned the association between the neutral cue and aversive sound, we calculated separate conditioned response scores for SCR magnitude from each phase (acquisition, acquisition avoid, extinction and extinction avoid). The conditioned response scores were the CS+ trials - the CStrials for each phase. A positive differential response score indicated a larger response for CS+ relative to CS-, indexing a conditioned response. We considered participants 'learners' if they displayed a positive differential response in any of the phases. Based on this criterion, only three participants out of the fifty participants displayed no differential responses in any of the phases. However, as removing

\footnotetext{
${ }^{1}$ We identified three non-responders in our sample based on the following criterion: participants who did not respond to more than $10 \%$ of trials. Removing these participants did not change the pattern or significance of results for SCR. Therefore, for reasons of completeness we decided to include these three participants in our analyses.
} 
these participants did not change the results reported here, for reasons of completeness we decided to include these three participants.

\section{Avoidance behaviour, ratings and SCR magnitude analysis}

The analysis was conducted using the mixed models procedure in SPSS 21.0 (SPSS, Inc; Chicago, Illinois). We conducted separate multilevel models on avoidance behaviour, ratings and SCR magnitude. For avoidance behaviour, we entered Stimulus (CS+, CS-) and Phase (Acquisition Avoid and Extinction Avoid) at level 1 and individual subjects at level 2. For behavioural ratings and SCR magnitude we entered Stimulus (CS+, CS-) and Phase (Acquisition, Acquisition Avoid, Extinction and Extinction Avoid) at level 1 and individual subjects at level 2. To assess the effect of extinction across time on SCR magnitude, we included a separate multilevel model, where we entered Stimulus (CS+, CS-) and Time (Early, Late) at level 1 and individual subjects at level 2. In all models, we used a diagonal covariance matrix for level 1. Random effects included a random intercept for each individual subject, where a variance components covariance structure was used. Fixed effects included Stimulus, Phase and Time. We used a maximum likelihood estimator for each multilevel model. We followed up with Least Square Difference pairwise comparisons. Furthermore, we did not apply a correction for multiple comparisons.

To assess specificity of total rate of avoidance's impact on extinction, we performed hierarchical regression analyses on the total rate of avoidance for each phase separately (acquisition avoid and extinction avoid) and the SCR magnitude differences scores from each phase. We entered SCR magnitude difference scores 
(CS+ - CS-) from acquisition, acquisition avoid and extinction avoid in the first step, and then SCR magnitude difference scores from extinction in the second step.

\section{Results}

Figure 2 about here

\section{Avoidance response}

During acquisition avoid the majority of participants avoided the CS+ at least once (37 out of 50; $M$ reinforcement rate $=36 \%, S D$ reinforcement rate $=10.9 \%$, range of reinforcement rate $=25-50 \%$ ).

A significant main effect of Stimulus, $F(1,197.323)=11.684, p=.001$ was found for avoidance responses (see Fig 2). Across both avoidance phases (i.e. acquisition and extinction avoidance), participants displayed more avoidance to the CS+ vs. CS-. There was no significant main effect of Phase, $F(1,197.323)=2.360, p$ $=.126$ or interaction between Stimulus and Phase, $F(1,197.323)=1.450, p=.230$ (for descriptive statistics see Table 1). However, a pairwise comparison revealed that there was a significant drop in avoidance responding to the CS+ from acquisition to extinction, $p=.001 .^{2}$

\footnotetext{
2 Despite the non-parametric distributions of the avoidance responses (see Figure 2), the results remain the same if we conduct non-parametric Friedman tests. For acquisition avoid, there were significantly more avoidance responses for CS+ versus CS-, Chi-square $=24.143, p<.001$. For extinction avoid, there were significantly more avoidance responses for CS+ versus CS-, Chi-square $=12, p=.001$. Furthermore, there were significantly more avoidance responses for CS+ during acquisition avoid, compared to extinction avoid, Chi-square $=4.840, p=.028$.
} 


\section{Ratings}

All subjects rated the sound stimulus (the US) as aversive $(M=1.92, S D=$ 1.01 , where $1=$ very negative and $9=$ very positive $)$ and arousing $(M=6.96, S D=$ 1.64 , where 1 = calm and $9=$ excited).

For the expectancy ratings, significant main effects of Stimulus, $F(1,308.947)$ $=353.024, p<.001$, and Phase, $F(3,160.667)=66.692, p<.001$ as well as an interaction between Stimulus and Phase emerged, $F(3,160.667)=32.422, p<.001$ (for descriptive statistics of ratings see Table 1 and Fig 3a). As expected, for all phases participants reported greater expectancy of the sound with the CS+, compared to the CS-, all p's <.001. Furthermore, participants rated greater expectancy of the sound with the CS+ vs. CS- during the earlier acquisition phases, compared to the later extinction phases, $p$ 's $<.05$. In particular, participants rated greater expectancy of the sound with the CS+ and less expectancy of the sound with the CS- during acquisition avoid, compared to acquisition, p's <.05. Additionally, participants rated greater expectancy of the sound with the CS+ during extinction, compared to extinction avoid, $p=.031$. No change in expectancy ratings of the sound with the CS- was observed between extinction and extinction avoid, $p=.455$.

\section{SCR magnitude}

A significant main effect of Stimulus, $F(1,325.927)=10.578, p=.001$ was found for SCR magnitudes (see Fig 3b). Across all phases, participants displayed larger SCR magnitudes to CS+ vs. CS-. In addition, there was a main effect of Phase, $F(3,185.584)=18.002, p<.001$, such that SCR magnitudes decreased across time. 
We observed no significant interaction between Stimulus and Phase in our omnibus test, $F(3,185.584)=.995, p=.397$ (for descriptive statistics see Table 1). Given our a-priori prediction that the conditioned response would change depending on the availability of avoidance and the main effects observed for Stimulus and Phase, we conducted pairwise comparisons to assess whether CS+ vs CSdifferences were significant in each phase. The acquisition and extinction phases showed significant differences in SCR magnitude between the CS+ and CS-, $p=$ .018 , and $p=.004$; no significant differences in SCR magnitude were observed between CS+ and CS- during acquisition avoid and extinction avoid, $p=.695$, and $p$ $=.216$.

During extinction, SCR magnitude remained larger for the CS+ versus the CS, $F(1,197.220)=9.015, p=.003$, and this difference in SCR magnitude did not decrease by Time, $F(1,197.220)=3.001, p=.085$ or Stimulus $x$ Time $F(1,197.220)=$ $.068, p=.794$.

Figure 3 about here.

\section{Total rate of avoidance for acquisition avoid and extinction avoid}

A hierarchical regression analysis confirmed that the total rate of avoidance during acquisition avoid specifically predicted the SCR extinction difference score, over SCR differences scores from acquisition, acquisition avoid, and extinction avoid; adding the extinction difference score in the second step significantly improved the model: first step: $R^{2}=.005, F(3,46)=.081, p=.970$, second step: $\Delta R^{2}=.104, F(1,45)=$ $5.256, p=.027$. 
A similar pattern was also observed for the total rate of avoidance during extinction avoid, which specifically predicted the SCR extinction difference score, over SCR differences scores from acquisition, acquisition avoid, and extinction avoid; adding the extinction difference score in the second step significantly improved the model: first step: $R^{2}=.074, F(3,46)=1.218, p=.314$, second step: $\Delta R^{2}=.115, F(1,45)=6.350, p=.015$.

Removing participants who never avoided during the experiment $(n=13)$ moved the hierarchical regression analyses to trend: the total rate of avoidance in acquisition avoid predicting extinction, $\Delta R^{2}=.085, F(1,45)=3.128, p=.087$; and the total rate of avoidance in extinction avoid predicting extinction, $\Delta R^{2}=.101, F(1,45)=$ 4.116, $p=.051$.

\section{Discussion}

In the current study, we examined how availability of avoidance interacts with the expression of learned threat. Within an acquisition phase where learned threat associations were being reinforced with an aversive sound, and avoidance was available some of the time, we found reduced differential skin conductance responses for unavoidable threat and safety cues. However, in an extinction phase where avoidance was unavailable and where learned threat associations were no longer being reinforced by an aversive sound, we found continued differential skin conductance responses for threat versus safety cues. Furthermore, we found individual differences in the total rate of avoidance during acquisition and extinction avoid phases to separately predict the extent of differential skin conductance response between learned threat versus safety cues during extinction, such that 
greater avoidance maintained a larger conditioned response during extinction. Our data suggest that availability of avoidance attenuates learned threat associations due to reduced US reinforcement. Removing the availability of avoidance during extinction, however, leaves the learned threat association to return; this effect is particularly noticeable for those individuals who engaged in more avoidant behaviour. These findings further our understanding of the role of threat avoidance on extinction resistance and highlight the potential of relevance of avoidance mechanisms in clinical disorders such as anxiety.

We included unavoidable trials as a novel manipulation to assess how availability of avoidance may alter the expression of learned threat. To our knowledge only one other study has included unavoidable trials within an avoidance context (Rattel et al. 2017). Overall, for both acquisition and extinction avoid phases, greater avoidance behaviour was observed for the learned threat versus safe cues. Avoidance behaviour to the learned threat dropped between the acquisition avoid to extinction avoid phases, albeit that the Stimulus $x$ Phase interaction in the omnibus test was not significant. Moreover, we found greater expectancy ratings for threat versus safe cues during acquisition avoid, compared to acquisition. However, expectancy ratings for threat versus safe cues were comparable during extinction and extinction avoid. This specific result suggests that participants learned to use avoidance adaptively, engaging in avoidance more during a phase with reinforcement of the US, compared to a phase without reinforcement of the US, similar to previous work (Vervliet \& Indekeu, 2015). Interestingly, we found this pattern of results despite there not being any explicit costs of avoidance (e.g. loss in money or time). However, a notable cost of avoidance would be not knowing if the 
reinforcement rate between the CS-US has changed. For example, during the extinction avoid phase withholding avoidance would be useful in determining whether the CS is safe or not.

We observed substantial variability in avoidance behaviour across individuals, as some never avoided, some always avoided, and others were more selective of avoiding when and what. Thirteen individuals never avoided, perhaps because they did not find the US particularly aversive or because they did not want to miss out on any information about the CS contingencies. Seven individuals always avoided (including the safe cue), suggesting that they may have overestimated the chance of threat or that they did not want to know about the CS contingencies. Given the substantial variability of avoidance behaviour within this study and others (e.g. Rattel et., 2017), large samples may be needed to examine what drives individual differences in avoidance behaviour.

Furthermore, we observed reduced skin conductance magnitude difference between unavoidable learned threat versus safety cues during phases with availability of avoidance, compared to the phases without availability of avoidance. This finding sits alongside recent work in rodents and humans, which show that active avoidance can attenuate learned threat associations (Baratta et al., 2007; Boeke et al., 2017; Cain \& LeDoux, 2007) and encourage feelings of relief (Andreatta et al., 2017; Vervliet et al., 2017). In these studies, learning how to actively avoid a shock facilitated extinction during the same avoidance learning context on the same day (Cain \& LeDoux, 2007), in the same avoidance context on the next day (Boeke et al., 2017), and in a different threat learning context days later (Baratta et al., 2007). It is possible that active avoidance leads to greater feelings of 
having control and therefore provides relief and reward. This finding may be particularly relevant for understanding avoidance behaviour in anxiety disorders. For example, the short-term relief experienced from availability of avoidance (e.g. safety behaviours) may be greater, and potentially rewarding in some anxious populations e.g. specific phobia. Indeed, a recent study showed that individuals with low distress tolerance displayed sustained feelings of relief over continuous omissions of threat by avoidance (Vervliet et al., 2017). Further work is needed to clarify the mechanisms underlying the role of avoidance and relief on learned threat responding.

In the extinction phase, we observed greater expectancy ratings and differential skin conductance responding to the learned threat versus safety cues. Across the extinction phase, the differential skin conductance response did not decrease over time. Furthermore, individuals who engaged in more avoidance during the acquisition and extinction avoid phases displayed the largest conditioned response during extinction. Both avoidance phases predicted extinction resistance. However, the avoidance phases may have predicted extinction resistance for different reasons. Avoidance during acquisition avoid is more likely to be predictive of extinction resistance, due to the temporal order of the phases; avoidance during extinction avoid is likely to have predicted extinction resistance, because it is highly correlated with avoidance during acquisition avoid. Individuals who engaged in more avoidance during acquisition avoid are likely to return to this behaviour after the extinction phase. Notably, the results for individual differences in the total rate of avoidance were weaker after removing individuals who never avoided, suggesting that the results were driven by these individuals. This is in line with previous 
research showing that engaging in avoidance behaviour reduces extinction (Lovibond et al., 2009; Rattel et al., 2017; Vervliet \& Indekeu, 2015; Xia et al., 2017). Essentially, our results support the notion that avoidance behaviour leaves the learned threat association unchallenged. Furthermore, this effect is particularly noticeable for those individuals who rely on avoidance more often. These results support those of same day extinction studies (Lovibond et al., 2009; Vervliet \& Indekeu, 2015; Xia et al., 2017) and not following day extinction studies (Boeke et al., 2017), suggesting there may be temporal differences in the effect of avoidance on extinction resistance. Taken together these findings provide further evidence for how pervasive avoidance behaviour may block extinction. This research may be particularly relevant for clinicians using current exposure-based treatments or aiming to develop novel treatments for anxiety disorder patients who engage in persistent avoidance behaviour. The next crucial steps for future research is to understand (1) how classic threat conditioning and instrumental threat conditioning interact to produce pervasive avoidance behaviour (LeDoux et al., 2017), and (2) how avoidance behaviour (and other safety behaviours) can be mitigated and active coping (facing fears and anxiety) can be encouraged, which will have important implications for clinical applications.

The design specifics of the current study should be further addressed in future research. A strength of the design was that we included unavoidable trials, similarly to Rattel et al. (2017), in order to assess how the expression of learned threat changed as a function avoidance availability. Future work should attempt to incorporate unavoidable trials to further understand how availability of avoidance alters the conditioned response. Notably, there were some weaknesses to the 
design. For example, the acquisition avoid phase follows the acquisition phase, which is a time confound. On this basis the reduction of the conditioned response could be an effect of, or interact with, habituation rather than lack of US reinforcement. In this experiment, we did not include a comparison group who underwent acquisition and extinction, without avoidance learning, therefore we cannot disentangle the effect of acquisition avoid on extinction. However, studies that have used between-group (Lovibond et al., 2009; Rattel et al., 2017) and withingroup designs (Vervliet \& Indekeu, 2015) have shown similar results of low-cost avoidance learning (i.e. taking shorter detours or pressing a button with little consequences) on extinction resistance. Another limitation of the experiment is that we did not acquire an explicit rating of the expectancy of the sound, relief felt, or contingency awareness on avoidable and unavoidable trials separately. Further work may be able to clarify how these aspects relate to skin conductance attenuation during avoidance, by acquiring online ratings of expectancy, relief and contingency awareness. Furthermore, the generality of the study findings should be tested in future studies using different: (1) unconditioned stimuli that vary in levels of aversiveness, (2) number of trials in extinction to assess how many trials are required to bypass extinction resistance from avoidance, (3) reinforcement rates, and (4) ratios between threat and safe cues. Furthermore, the sample contains mainly female participants, and future studies should more carefully balance their sample in terms of gender.

In conclusion, the results here show a critical role of avoidance availability, as availability attenuates the conditioned response but unavailability recovers the conditioned response, prolonging threat extinction, particularly if avoidant behaviour 
is pervasive. Importantly, these results highlight an opportunity for further research to explore the role of avoidance in: (1) providing short term relief and, (2) extinction resistance in anxiety disorder pathology and clinical treatment. 


\section{References}

Andreatta, M., Michelmann, S., Pauli, P., \& Hewig, J. (2017). Learning processes underlying avoidance of negative outcomes. Psychophysiology, 54(4), 578590.

Baratta, M., Christianson, J., Gomez, D., Zarza, C., Amat, J., Masini, C., . . Maier, S. (2007). Controllable versus uncontrollable stressors bi-directionally modulate conditioned but not innate fear. Neuroscience, 146(4), 1495-1503.

Boeke, E. A., Moscarello, J. M., LeDoux, J. E., Phelps, E. A., \& Hartley, C. A. (2017). Active avoidance: Neural mechanisms and attenuation of Pavlovian conditioned responding. Journal of Neuroscience, 37(18), 4808-4818.

Boyle, S., Roche, B., Dymond, S., \& Hermans, D. (2015). Generalisation of fear and avoidance along a semantic continuum. Cognition and Emotion(ahead-ofprint), 1-13.

Cain, C. K., \& LeDoux, J. E. (2007). Escape from fear: a detailed behavioral analysis of two atypical responses reinforced by CS termination. Journal of Experimental Psychology: Animal Behavior Processes, 33(4), 451.

Delgado, M. R., Jou, R. L., LeDoux, J. E., \& Phelps, E. A. (2009). Avoiding negative outcomes: tracking the mechanisms of avoidance learning in humans during fear conditioning. Neuroeconomics, 72.

Dymond, S., \& Roche, B. (2009). A contemporary behavioral analysis of anxiety and avoidance. The Behavior Analyst, 32, 7-28.

Jenkins, W. O., \& Stanley Jr, J. C. (1950). Partial reinforcement: a review and critique. Psychological Bulletin, 47(3), 193. 
Krypotos, A.-M., Effting, M., Kindt, M., \& Beckers, T. (2015). Avoidance learning: a review of theoretical models and recent developments. Frontiers in Behavioral Neuroscience, 9, 189.

LeDoux, J., Moscarello, J., Sears, R., \& Campese, V. (2017). The birth, death and resurrection of avoidance: a reconceptualization of a troubled paradigm. Molecular Psychiatry, 22(1), 24-36.

Lommen, M. J., Engelhard, I. M., \& van den Hout, M. A. (2010). Neuroticism and avoidance of ambiguous stimuli: Better safe than sorry? Personality and Individual Differences, 49(8), 1001-1006.

Lovibond, P. F., Mitchell, C. J., Minard, E., Brady, A., \& Menzies, R. G. (2009). Safety behaviours preserve threat beliefs: protection from extinction of human fear conditioning by an avoidance response. Behaviour Research and Therapy, 47(8), 716-720.

Lovibond, P. F., Saunders, J. C., Weidemann, G., \& Mitchell, C. J. (2008). Evidence for expectancy as a mediator of avoidance and anxiety in a laboratory model of human avoidance learning. The Quarterly Journal of Experimental Psychology, 61(8), 1199-1216.

Milad, M. R., \& Quirk, G. J. (2012). Fear extinction as a model for translational neuroscience: ten years of progress. Annual review of psychology, 63, 129151.

Morriss, J., Christakou, A., \& Van Reekum, C. M. (2015). Intolerance of uncertainty predicts fear extinction in amygdala-ventromedial prefrontal cortical circuitry. Biology of Mood \& Anxiety Disorders, 5(1), 1. 
Morriss, J., Christakou, A., \& Van Reekum, C. M. (2016). Nothing is safe: Intolerance of uncertainty is associated with compromised fear extinction learning. Biological Psychology, 121, 187-193.

Morriss, J., Macdonald, B., \& van Reekum, C. M. (2016). What Is Going On Around Here? Intolerance of Uncertainty Predicts Threat Generalization. PloS one, 11(5), e0154494.

Mowrer, O. H. (1951). Two-factor learning theory: summary and comment. Psychological Review, 58(5), 350.

Mowrer, O. H. (1956). Two-factor learning theory reconsidered, with special reference to secondary reinforcement and the concept of habit. Psychological Review, 63(2), 114.

Pittig, A., Brand, M., Pawlikowski, M., \& Alpers, G. W. (2014). The cost of fear: avoidant decision making in a spider gambling task. Journal of Anxiety Disorders, 28(3), 326-334.

Rattel, J. A., Miedl, S. F., Blechert, J., \& Wilhelm, F. H. (2017). Higher threat avoidance costs reduce avoidance behaviour which in turn promotes fear extinction in humans. Behaviour Research and Therapy, 96, 37-46.

van Meurs, B., Wiggert, N., Wicker, I., \& Lissek, S. (2014). Maladaptive behavioral consequences of conditioned fear-generalization: a pronounced, yet sparsely studied, feature of anxiety pathology. Behaviour Research and Therapy, 57, 29-37.

Vervliet, B., \& Indekeu, E. (2015). Low-cost avoidance behaviors are resistant to fear extinction in humans. Frontiers in Behavioral Neuroscience, 9. 
Vervliet, B., Lange, I., \& Milad, M. R. (2017). Temporal dynamics of relief in avoidance conditioning and fear extinction: Experimental validation and clinical relevance. Behaviour Research and Therapy, 96, 66-78.

Xia, W., Dymond, S., Lloyd, K., \& Vervliet, B. (2017). Partial reinforcement of avoidance and resistance to extinction in humans. Behaviour Research and Therapy, 96, 79-89. 


\section{Acknowledgements}

This research was supported by the Centre for Integrative Neuroscience and Neurodynamics (CINN) at the University of Reading. The authors thank the participants who took part in this study. For access to the data please contact Dr. Jayne Morriss. 
Table 1.

Summary of means (SD) for each dependent measure as a function of condition during each phase

\begin{tabular}{|c|c|c|c|c|c|c|c|c|}
\hline \multirow[b]{2}{*}{ Measure } & \multicolumn{6}{|c|}{ Acquisition } & \multicolumn{2}{|c|}{$\begin{array}{c}\text { Extinction } \\
\text { Avoid }\end{array}$} \\
\hline & $\mathrm{CS}+$ & CS- & CS+ & CS- & $\mathrm{CS}+$ & CS- & $\mathrm{CS}+$ & CS- \\
\hline Avoidance fr & ency & & $\begin{array}{c}3.35 \\
(2.55)\end{array}$ & $\begin{array}{c}1.68 \\
(2.43)\end{array}$ & & & $\begin{array}{c}2.36 \\
(2.82)\end{array}$ & $\begin{array}{c}1.56 \\
(2.51)\end{array}$ \\
\hline $\begin{array}{l}\text { Expectancy } \\
\text { rating }\end{array}$ & $\begin{array}{c}6.48 \\
(2.05)\end{array}$ & $\begin{array}{c}2.68 \\
(2.04)\end{array}$ & $\begin{array}{c}7.26 \\
(1.58)\end{array}$ & $\begin{array}{c}1.82 \\
(1.55)\end{array}$ & $\begin{array}{c}3.64 \\
(2.31)\end{array}$ & $\begin{array}{r}1.40 \\
(.90)\end{array}$ & $\begin{array}{r}2.70 \\
(2.03)\end{array}$ & $\begin{array}{l}1.28 \\
(.70)\end{array}$ \\
\hline $\begin{array}{l}\text { SCR } \\
\text { magnitude } \\
(\sqrt{\mu S})\end{array}$ & $\begin{array}{c}0.45 \\
(0.60)\end{array}$ & $\begin{array}{c}0.20 \\
(0.43)\end{array}$ & $\begin{array}{c}0.29 \\
(0.38)\end{array}$ & $\begin{array}{l}-0.03 \\
(0.44)\end{array}$ & $\begin{array}{l}-0.01 \\
(0.28)\end{array}$ & $\begin{array}{l}-0.17 \\
(0.27)\end{array}$ & $\begin{array}{l}-0.03 \\
(0.43)\end{array}$ & $\begin{array}{l}-0.15 \\
(0.53)\end{array}$ \\
\hline
\end{tabular}

Note: Avoidance frequency; Expectancy rating, 1 = 'Don't expect' - 9 'Do expect'; SCR magnitude $(\sqrt{ } \mu S)$, square root transformed and z-scored skin conductance magnitude measured in microSiemens 


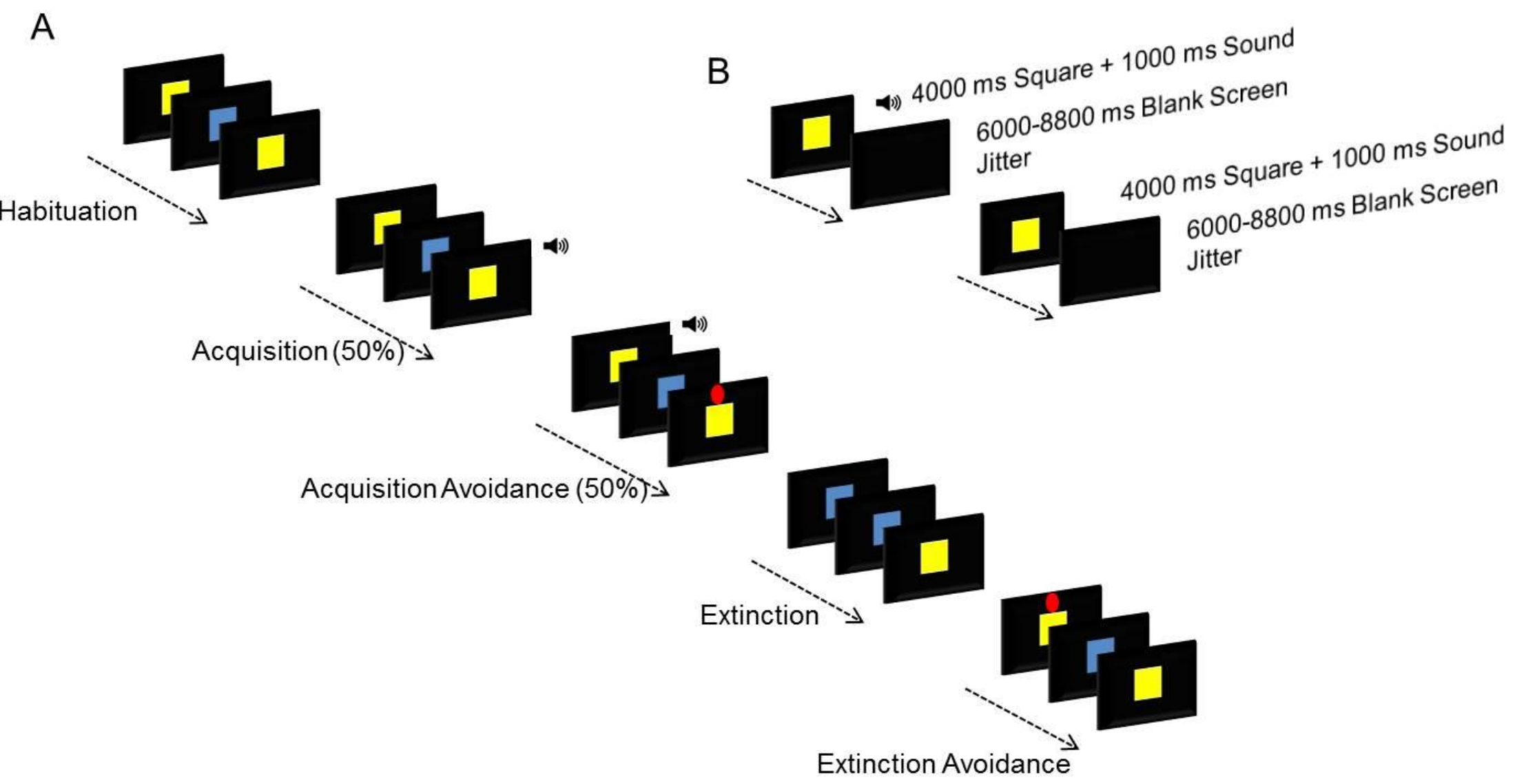

Fig 1. Image depicting (A) the experimental phases of the experiment, and (B) examples of unavoidable and avoidable trials. On avoidable trials a red dot would appear above the square. The red dot signified that the trial could be avoided by clicking the left mouse button which would move the experiment on to the next trial. 


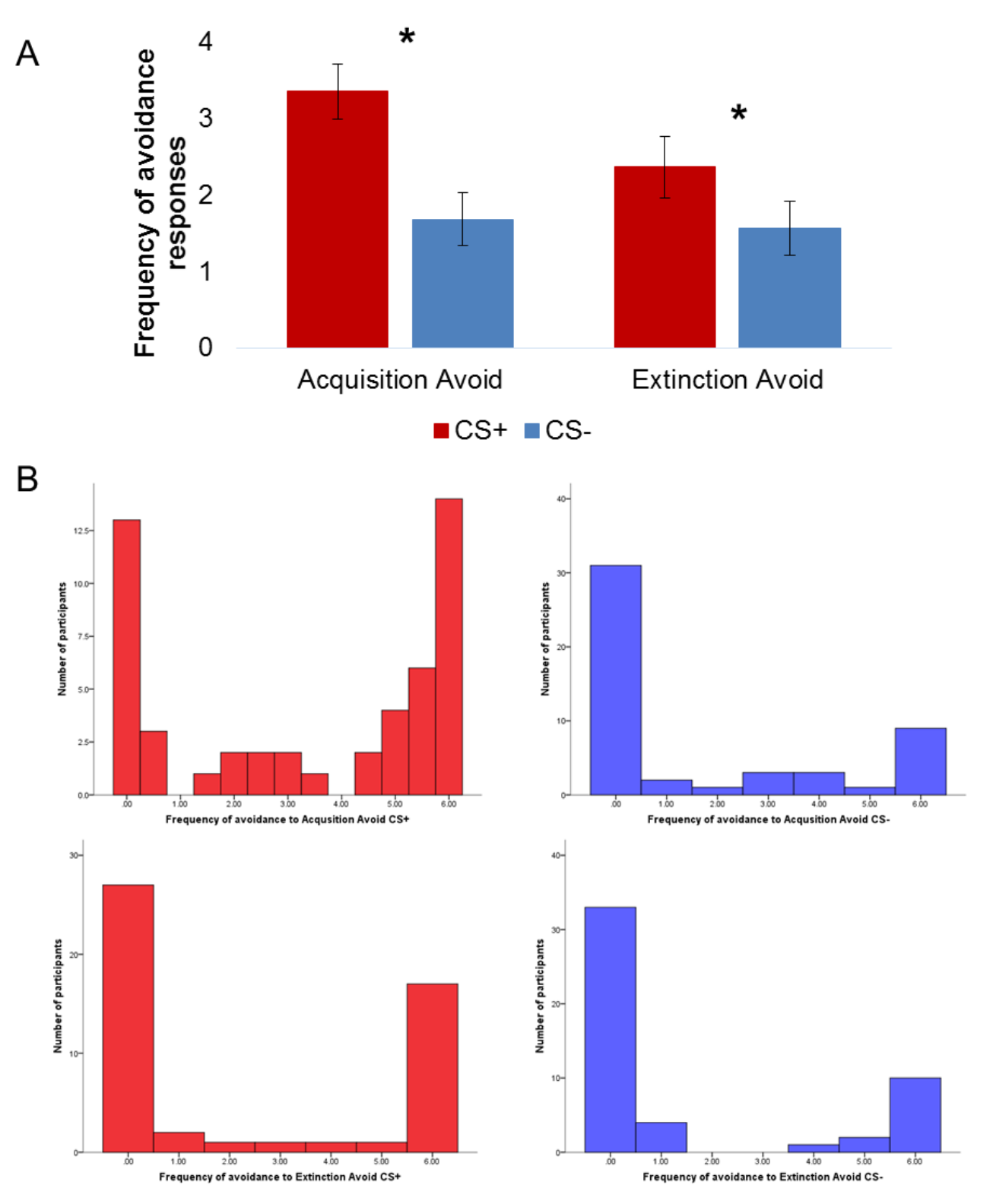

Fig 2. (A) Bar graph depicting frequency of avoidance responses during the acquisition and extinction avoid phases. Greater avoidance behaviour to the CS+ versus CS- was observed within acquisition and extinction avoid phases. Max frequency of avoidance responses was 6 per phase. (B) Histograms of the distribution of avoidance behaviour for each condition. Bars represent standard error of the mean. Asterisks represent significant differences between CS+ versus CS- conditions, $p<$ .05 . 
A 9

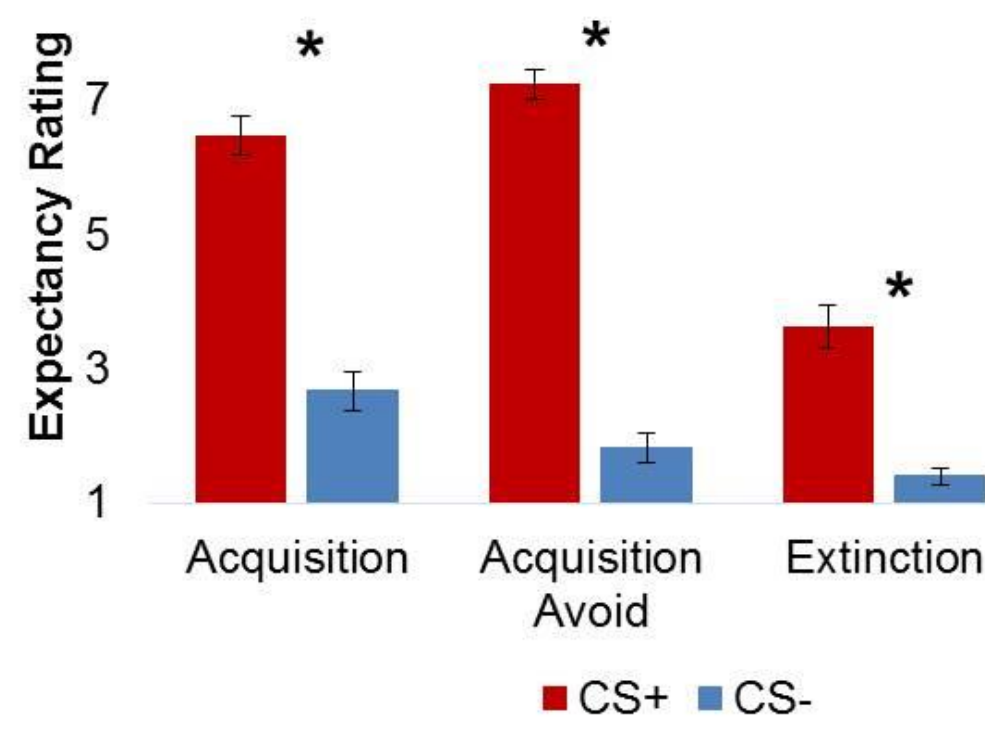

B

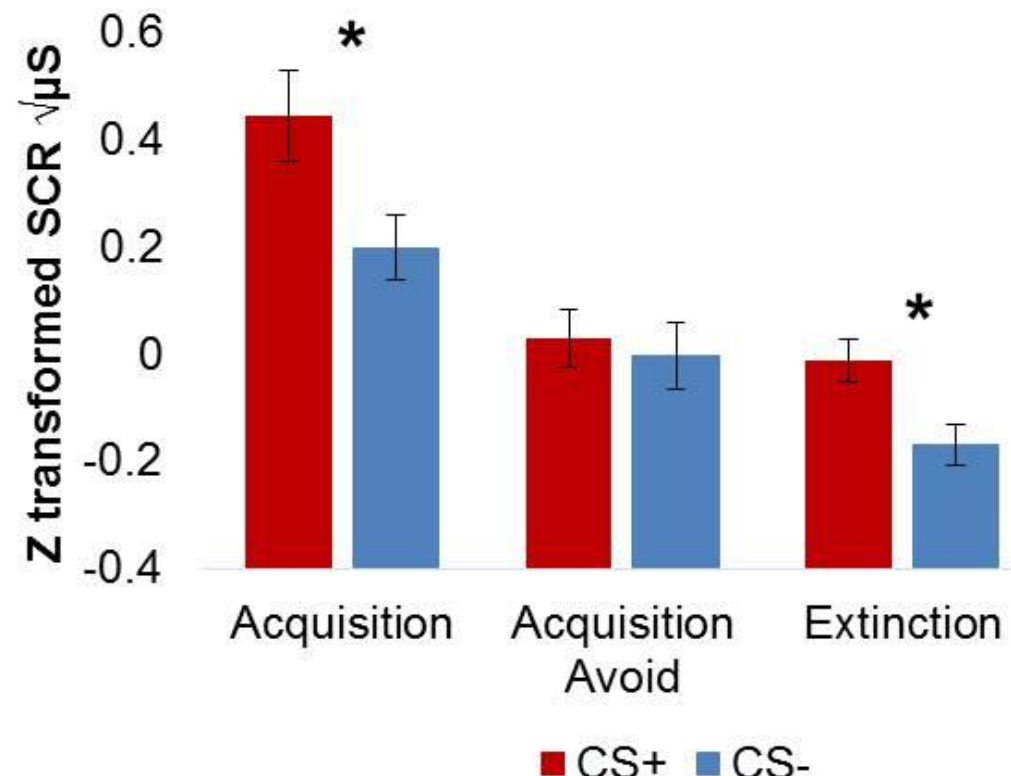

Fig 3. Bar graphs displaying (A) expectancy ratings and (B) SCR magnitude scores to the CS+ and CSduring all phases of the experiment. For all phases participants reported greater expectancy of the sound with the CS+, compared to the CS-. In addition, larger SCR magnitude responses were found for the CS+ versus CS- during acquisition and extinction, but not during the avoidance phases. Please note that the SCRs are averages from the unavoidable trials only and the expectancy ratings are averages from all trials. Bars represent standard error of the mean. Asterisks represent significant difference between CS+ versus CS- conditions, $p<.05$. Expectancy rating, 1 'Don't expect - 9 'Do expect'. SCR magnitude $({ } \mu S)$, square root transformed and $z$ scored skin conductance magnitude measured in microSiemens. 